\title{
A Remark on the Measurability of Large Games*
}

\author{
Guilherme Carmona ${ }^{\dagger}$
}

October 26, 2007

\begin{abstract}
A game with a continuum of players is described by a function assigning payoff functions to players and satisfying some measurability properties. In this note we establish the equivalence between several measurability assumptions that have been made in the literature.
\end{abstract}

Journal of Economic Literature Classification Numbers: C72.

Keywords: Games with a continuum of players, measurability.

\section{Introduction}

The study of games with a continuum of players was initiated by Schmeidler (1973). His formalization of such games parallels that of normal form games with a finite number of players given in Nash (1950) insofar as a payoff function is assigned to every player in the game.

In the presence of a continuum of players, the way that payoff functions are assigned to players must satisfy some measurability properties in order for the game to be amendable to analysis.

${ }^{*}$ I thank an anonymous referee for very helpful comments and John Huffstot for editorial assistance. Financial support from a Nova Fórum grant is gratefully acknowledged.

${ }^{\dagger}$ Address: Universidade Nova de Lisboa, Faculdade de Economia, Campus de Campolide, 1099032 Lisboa, Portugal; Phone: (351) 21380 1671; Fax: (351) 21387 0933; email: gcarmona@fe.unl.pt. 
However, several measurability assumptions have been made in the literature. The goal of this note is to establish that many of these assumptions are equivalent, therefore unifying apparently distinct results.

\section{Definitions and Main Result}

We consider games with a continuum of players in which each player has a separable metric space of actions and a continuous payoff function that depends only on his choice and on the distribution of actions. ${ }^{1}$ The set of players is represented by an atomless probability space $(T, \Xi, \varphi)$.

The set of actions is denoted by $A$, and we assume that it is a non-empty, separable metric space. By a distribution of actions we mean a Borel probability measure on $A$. Let $\mathcal{P}(A)$ be the space of Borel probability measures on $A$ endowed with the weak* topology (see Aliprantis and Border (1999, Chapter 14)). By Aliprantis and Border (1999, Theorem 14.12), it follows that $\mathcal{P}(A)$ is a compact metric space. We endow both $A$ and $\mathcal{P}(A)$ with the Borel $\sigma$-algebra induced by the corresponding topology.

Let $\mathcal{U}$ denote the space of real-valued continuous payoff functions defined on $A \times$ $\mathcal{P}(A)$. The set $\mathcal{U}$ represents the space of players' characteristics. We endow it with the supremum norm, thus making it a complete and separable metric space, and with the corresponding Borel $\sigma$-algebra.

A game with a continuum of players is defined by assigning a payoff function to each player. Thus, it is characterized by a function $U: T \rightarrow \mathcal{U}$ satisfying some measurability property.

Several assumptions can be made regarding the measurability of $U$ : (1) $U$ is measurable (Rath (1995), Khan, Rath, and Sun (1997), Khan, Rath, and Sun (2006)) (2) the function $(t, a, \tau) \mapsto g(t)(a, \tau)$ is measurable, (3) the function $(t, a) \mapsto g(t)(a, \tau)$ is measurable for all $\tau \in \mathcal{P}(A)$ (Schmeidler (1973), Rath (1992), Balder (2002)), and

\footnotetext{
${ }^{1}$ Games with a continuum of players and a separable, but not necessarily compact, metric space of actions have been consider in Khan and Sun (1995), Carmona (2007) and Carmona and Podczeck (2007).
} 
(4) the function $t \mapsto g(t)(a, \tau)$ is measurable for all $a \in A$ and $\tau \in \mathcal{P}(A)$.

Intuitively, these assumptions are ordered in terms of their strength, with (1) representing the strongest and (4) the weakest one. Proposition 1 strengthens this relationship by showing that all the above assumptions are equivalent.

Proposition 1 Let $g$ be a function from $T$ into $\mathcal{U}$ such that $g(T)$ is separable. Then, the following conditions are equivalent:

(1) $g$ is measurable.

(2) The function $(t, a, \tau) \mapsto g(t)(a, \tau)$ is measurable.

(3) The function $(t, a) \mapsto g(t)(a, \tau)$ is measurable for all $\tau \in \mathcal{P}(A)$.

(4) The function $t \mapsto g(t)(a, \tau)$ is measurable for all $a \in A$ and $\tau \in \mathcal{P}(A)$.

Some remark are in place before we turn to the proof of Proposition 1. First, Proposition 1 also holds when $(T, \Xi, \varphi)$ is complete and there exists $T^{\prime} \subseteq T$ such that $\varphi\left(T^{\prime}\right)=0$ and $g\left(T \backslash T^{\prime}\right)$ is separable. This implies that the probability distribution $\varphi \circ g^{-1}$ on $\mathcal{U}$ has a separable support, the importance of which has been highlighted by Khan (1989) and Rath (1996). Second, note that (4) implies that $(t, a, \tau) \mapsto g(t)(a, \tau)$ is a Carathéodory function, and hence measurable, i.e., (2) holds (see Aliprantis and Border (1999, Lemma 4.50)). Third, it has been shown in Rath (1992, Remark 7) that (1) implies (3). ${ }^{2}$ Forth, when $A$ is compact, the equivalence between (1) and (4) follows from Aliprantis and Border (1999, Theorem 4.54). Fifth, Rath (1992, Theorem 2) establishes the existence of a Nash equilibrium under the measurability condition (1). It is remarked there that the result can also be established under (3) using Lemma III.39 of Castaing and Valadier (1977). However, since (1) and (3) are equivalent, this lemma is not required and the proof of Theorem 2 in Rath (1992) applies verbatim to any of the measurability conditions considered here.

Our proof of Proposition 1 relies on a generalization of Theorem 4.54 in Aliprantis and Border (1999).

\footnotetext{
${ }^{2}$ Rath (1995) assumes that $A$ is compact, but his proof extends to the case when $A$ is an arbitrary metric space.
} 
Proposition 2 Let $(S, \Sigma)$ be a measurable space, $X$ a separable metric space, $(Y, d)$ a metric space and $C(X, Y)$, the space of continuous functions from $X$ to $Y$, be endowed with the metric $\delta(f, h)=\sup _{x \in X} d(f(x), h(x))$. If $g$ is a function from $S$ to $C(X, Y)$ such that $g(S)$ is separable and $s \mapsto g(s)(x)$ is measurable for all $x \in X$, then $g$ is measurable.

Proof. Since $g(S) \subseteq C(X, Y)$ is separable, $Z:=\overline{g(S)}$ is measurable and separable. Then, the class $\mathcal{C}=\left\{C_{\varepsilon}(f) \subseteq Z: \varepsilon>0\right.$ and $\left.f \in Z\right\}$ of closed balls generates the Borel $\sigma$-algebra of $Z$. Indeed, if $C$ is a closed subset of $Z$ and $D=\left\{f_{n}\right\}_{n=1}^{\infty} \subseteq C$ is countable and dense in $C$, then $C=\cap_{k=1}^{\infty} \cup_{n=1}^{\infty} C_{1 / k}\left(f_{n}\right)$.

As a result of the above, it follows that it is enough to show that $g^{-1}\left(C_{\varepsilon}(f)\right)$ is measurable for all $\varepsilon>0$ and $f \in Z$ (see Aliprantis and Border (1999, Corollary 4.23)). Let $\varepsilon>0$ and $f \in Z$ be arbitrary. Then,

$$
\begin{aligned}
& g^{-1}\left(C_{\varepsilon}(f)\right)=\{s \in S: \delta(g(s), f) \leq \varepsilon\}=\{s \in S: d(g(s)(x), f(x)) \leq \varepsilon \text { for all } x \in X\} \\
& =\cap_{k=1}^{\infty}\left\{s \in S: d\left(g(s)\left(x_{k}\right), f\left(x_{k}\right)\right) \leq \varepsilon\right\}
\end{aligned}
$$

where $\left\{x_{k}\right\}_{k}$ is a dense subset of $X$. Since $\left\{s \in S: d\left(g(s)\left(x_{k}\right), f\left(x_{k}\right)\right) \leq \varepsilon\right\}$ is measurable for all $k$, it follows that $g^{-1}\left(C_{\varepsilon}(f)\right)$ is measurable.

We conclude with the proof of Proposition 1.

Proof of Proposition 1. We show that (1) implies (2), (2) implies (3), (3) implies (4) and (4) implies (1).

In order to show that (1) implies (2), define $G: T \times A \times \mathcal{P}(A) \rightarrow \mathcal{U} \times A \times \mathcal{P}(A)$ by $G(t, a, \tau)=(g(t), a, \tau)$ and $\pi: \mathcal{U} \times A \times \mathcal{P}(A) \rightarrow \mathbb{R}$ by $\pi(u, a, \tau)=u(a, \tau)$ for all $t \in T, a \in A, \tau \in \mathcal{P}(A)$ and $u \in \mathcal{U}$. Then, $\pi$ is continuous and $G$ is measurable, implying that the function $(t, a, \tau) \mapsto g(t)(a, \tau)$ is measurable since it equals $\pi \circ G$.

We next show that (2) implies (3). Let $\tau \in \mathcal{P}(A)$. Define $\alpha: T \times A \rightarrow T \times A \times \mathcal{P}(A)$ by $\alpha(t, a)=(t, a, \tau)$ and $\beta: T \times A \times \mathcal{P}(A) \rightarrow \mathbb{R}$ by $\beta(t, a, \nu)=g(t)(a, \nu)$ for all $t \in T$, $a \in A$ and $\nu \in \mathcal{P}(A)$. Then, the function $(t, a) \mapsto g(t)(a, \tau)$ is measurable since it equals $\beta \circ \alpha$.

We can show that (3) implies (4) in a similar way. Let $a \in A$ and $\tau \in \mathcal{P}(A)$. Define $\gamma: T \rightarrow T \times A$ by $\gamma(t)=(t, a)$ and $\rho: T \times A \rightarrow \mathbb{R}$ by $\rho(t, x)=g(t)(x, \tau)$ for 
all $t \in T$ and $x \in A$. Then, the function $t \mapsto g(t)(a, \tau)$ is measurable since it equals $\rho \circ \gamma$.

Finally, it follows from Proposition 2 that (4) implies (1).

\section{References}

Aliprantis, C., And K. Border (1999): Infinite Dimensional Analysis. Springer, Berlin.

BALDER, E. (2002): "A Unifying Pair of Cournot-Nash Equilibrium Existence Results," Journal of Economic Theory, 102, 437-470.

Carmona, G. (2007): "Large Games with Countable Characteristics," forthcoming, Journal of Mathematical Economics.

Carmona, G., and K. Podczeck (2007): "On the Existence of Pure Strategy Equilibria in Large Games," Universidade Nova de Lisboa and Universität Wien.

Castaing, C., And M. Valadier (1977): Convex Analysis and Measurable Multifunctions. Springer, Berlin.

Khan, M. A. (1989): "On Cournot-Nash Equilibrium Distributions For Games with a Nonmetrizable Action Space and Upper Semicontinuous Payoffs," Transactions of the American Mathematical Society, 315, 127-146.

Khan, M. A., K. Rath, and Y. Sun (1997): "On the Existence of Pure Strategy Equilibria in Games with a Continuum of Players," Journal of Economic Theory, $76,13-46$.

_ (2006): "The Dvoretzky-Wald-Wolfowitz Theorem and Purification in Atomless Finite-Action Games," International Journal of Game Theory, 34, 91-104.

Khan, M. A., And Y. Sun (1995): "Extremal Structures and Symmetric Equilibria with Countable Actions," Journal of Mathematical Economics, 24, 239-248. 
NAsh, J. (1950): "Non-Cooperative Games," Ph.D. thesis, Princeton University.

RAth, K. (1992): "A Direct Proof of The Existence of Pure Strategy Equilibria in Games with a Continuum of Players," Economic Theory, 2, 427-433.

— (1995): "Representation of Finite Action Large Games," International Journal of Game Theory, 24, 23-35.

— (1996): "Existence and Upper Hemicontinuity of Equilibrium Distributions of Anonymous Games with Discontinuous Payoffs," Journal of Mathematical Economics, 26, 305-324.

SchmeIdler, D. (1973): "Equilibrium Points of Nonatomic Games," Journal of Statistical Physics, 4, 295-300. 